\title{
Plasma Level of Umbilical Cord Hemeoxygenase-1 (HO-1) and Neonatal Outcome in Early Onset and Late Onset Severe Preeclampsia
}

\author{
Muhammad Ilham Aldika Akbar ${ }^{1,2}$, Indah Mayang Sari ${ }^{1}$, Ernawati ${ }^{1,2}$, Aditiawarman $^{1}$ \\ ${ }^{1}$ Department of Obstetric and Gynecology, Faculty of Medicine, Universitas Airlangga/Dr. Soetomo General Hospital, \\ Surabaya, Indonesia \\ ${ }^{2}$ Department of Obstetric and Gynecology, Faculty of Medicine, Universitas Airlangga/Universitas Airlangga Hospital, \\ Surabaya, Indonesia
}

Background: Many studies had discovered that early onset severe preeclampsia (EO-PE) has worst maternal and neonatal outcome compared to late-onset type (LO-PE), related to its placental involvement. Severe preeclampsia was defined as newly onset severe hypertension developed after 20 weeks gestation in previously normal blood pressure women, with coexistence of proteinuria, or maternal organ or uteroplacental dysfunction. Hemeoxygenase-1 (HO-1) is an enzyme with multiple effect which is protective to pregnancy.

Materials and Methods: The total study subjects were 40 pregnant women consisted of 10 EO-PE, 10 normal early onset pregnancy (EO-NP), 10 LO-PE, and 10 normal late onset pregnancy (LO-NP). As much as $5 \mathrm{cc}$ of plasma from umbilical cord was taken as soon as the baby was born, and the HO-1 level was examined by enzyme-linked immunosorbent assay (ELISA). The primary outcome were umbilical cord HO-1 level and neonatal composite morbidity (low Apgar score, low birthweight, length of stay $>5$ day, respiratory distress syndrome, jaundice and neonatal death).

Results: The plasma level of HO-1 in EO-PE subjects were lower than EO-NP $(0.96 \pm 0.37 \mathrm{ng} / \mathrm{mL} v \mathrm{~s} .2 .43 \pm 0.58 \mathrm{ng} / \mathrm{mL}$, $p<0.001)$. There were no significant differences in the level of HO-1 in LO-PE and LO-NP $(2.18 \pm 1.07 \mathrm{ng} / \mathrm{mL} v$ s. $3.02 \pm 0.64 \mathrm{ng} /$ $\mathrm{mL}, p=0.277)$. Plasma level of umbilical cord HO-1 of EO-PE patients was lower compared to LO-PE $(0.96 \pm 0.37 \mathrm{ng} / \mathrm{mL} v \mathrm{v}$. $2.18 \pm 1.07 \mathrm{ng} / \mathrm{mL}, p=0.034)$. Neonatal outcome of EO-PE was worse than EO-NP $(p=0.033)$, and LO-PE $(p=0.003)$, while in LO-PE did not different with LO-NP $(p=0.211)$.

Conclusion: EO-PE is associated with lower plasma umbilical cord level of HO-1 and worse neonatal outcome compared to LO-PE. This indicating abnormal placental blood vessel development, placental ischemia in EO-PE, lead to reduced uteroplacental perfusion and significantly worse neonatal outcome compared to LO-PE.

Keywords: severe preeclampsia, early onset preeclampsia, late onset preeclampsia, hemeoxygenase-1

Date of submission: January 8, 2019

Last Revised: February 11, 2019

Accepted for publication: February 12, 2019

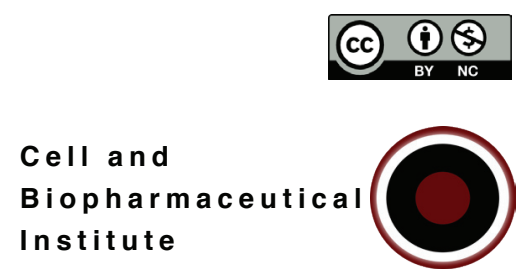

Corresponding Author:

Muhammad Ilham Aldika Akbar

Department of Obstetric and Gynecology

Faculty of Medicine, Universitas Airlangga/Dr. Soetomo General Hospital

Jl. Airlangga No.4-6, Surabaya, Indonesia

E-mail: muhammad-i-a-a@fk.unair.ac.id

\section{Biopharmaceutical Institute}




\section{Introduction}

Preeclampsia is a major contributor of maternal and fetal morbidity and mortality worldwide. ${ }^{1}$ Recently preeclampsia is classified based on the onset of the disease into early onset preeclampsia (EO-PE) $<34$ week gestation and late onset preeclampsia (LO-PE) $>34$ week. These two type of preeclampsia were suspected to have a different pathophysiology, clinical manifestation and outcome.,.$^{2,3}$ EO-PE occurs mainly due to abnormal placentation which can be caused by reduced of cell protective factors (such as nitrite, hemeoxygenase-1 (HO-1)), or oxidative stress, genetic, environmental and immunological factors. ${ }^{2,4}$ Whereas LO-PE is associated with underlying maternal disease coexisted in pregnancy, rather than abnormal placental development. ${ }^{2,3}$

Earlier study mentioned that there were two protective pathways in pregnancy, namely the hemeoxygenase-carbon monoxide/HO-CO pathway and the cysthathione- $\gamma$-lyase pathway. ${ }^{4}$ Deficiency in this protective pathway will lead to abnormal development of placental vascular system, eventually ended in preeclampsia. HO-1 is an enzyme that degrades free heme to produce a number of carbon monoxide (CO), iron $\left(\mathrm{Fe}^{2+}\right)$, and biliverdin through cytochrome P450 reductase (CPR) ${ }^{4,5}$ The in vivo and in vitro studies showed that HO-1 functions as a cytoprotector by degrading free heme which is highly reactive cytotoxic substance, and produces $\mathrm{CO}$ as a mediator of inhibiting inflammatory and apoptotic processes. ${ }^{4} \mathrm{HO}-1$ is expressed in the syncytiotrophoblast and involved in placental cell protection and relaxation of blood vessels during pregnancy. ${ }^{6}$ In preeclampsia, there is a disruption of regulation and development of placental blood vessels which is thought to be caused by reduced expression of HO-1 in the placenta. In this study we measure plasma HO-1 levels of umbilical cord and fetal outcome in EO$\mathrm{PE}, \mathrm{LO}-\mathrm{PE}$ and normal pregnancy, to determine the role of placental HO-1 in the pathogenesis of preeclampsia and its relation to the clinical outcome.

\section{Materials and methods}

This is a comparative analytic observational study with cross sectional method, comparing HO-1 levels of umbilical cord blood of newborns and neonatal outcome in EO-PE, LO$\mathrm{PE}$ and control groups (early onset-normal preganncy (EONP) and late onset-normal pregnancy (LO-NP)). This study was conducted in Dr. Soetomo General Hospital, Airlangga University Hospital and Dr. M. Soewandhi Hospital,
Surabaya, during December 2016 - February 2017. Inclusion criteria were pregnant women with severe preeclampsia and normal pregnant women $>20$ weeks. Severe preeclampsia was diagnosed based on the International Society for The Study of Hypertension in Pregnancy (ISSHP) classification: severe hypertension (blood pressure $>160 / 110 \mathrm{mmHg}$ ) developing after 20 weeks gestation and the coexistence of one of this condition (proteinuria, maternal organ dysfunction, or uteroplacental dysfunction). Maternal organ dysfunction involved: renal insufficiency (serum creatinine $>90 \mathrm{umol} / \mathrm{L}$ ), liver involvement (elevated transaminase, severe right upper quadrant or epigastric pain), neurological complication (eclampsia, altered mental status, blindness, stroke, hyperreflexia, etc.), and haematological complication. ${ }^{7}$ While the exclusion criteria were pregnant women who had an co-existing diseases (such as metabolic diseases, systemic diseases, autoimmune, gynecological infections and tumors), refuse to be involved in the research, smokers and multiple pregnancies. The sample size of each group was 10 patients, based on previous related study. ${ }^{8}$ The sampling in severe preeclampsia groups was done by consecutive sampling method, while in control group was carried out using a simple randomized method. As much as $5 \mathrm{cc}$ of plasma umbilical cord was taken as soon as the baby is born and then HO-1 levels were examined by enzymelinked immunosorbent assay (ELISA) (StressXpress ELISA kit, StressMarq Biosciences Inc, Victoria, Canada). Babies were followed until they were discharged from the hospital, and neonatal composite morbidity was assessed. Neonatal composite morbidity consisted of: Apgar score $<3$, baby birthweight $<2000$ gram, neonatal length of stay $>5$ days, respiratory distress syndrome, jaundice and neonatal death. Apgar score was measured of the fetal clinical performance on $1^{\text {st }}$ minute, based on appearance, pulse, grimace, activity, and respiratory. ${ }^{9}$ Respiratory distress syndrome was diagnosed based on clinical sign of dyspnea, grunting, tachypnea (respiratory rate $>60 \mathrm{x} /$ minutes), chest indrawing, cyanosis, laboratory, and chest-x ray. Data was analyzed using Anova test, post hoc Tamhane test, Kruskal Wallis test, Fisher exact test, and eta association test to evaluate the different and the associatioan between each variable (SPSS 23, SPSS Inc., Illinois, USA).

\section{Results}

\section{Characteristics of Research Samples}

Based on the data in Table 1, we found a significant differences in the age of the patients between EO-PE vs. 
Table 1. General characteristics.

\begin{tabular}{|c|c|c|c|c|}
\hline & EO-NP $(n=10)$ & EO-PE $(n=10)$ & LO-NP (n=10) & LO-PE (n=10) \\
\hline Variables & $\begin{array}{l}\text { n / Mean } \pm \text { SD / } \\
\text { Median (range) }\end{array}$ & $\begin{array}{l}\text { n / Mean } \pm \text { SD / } \\
\text { Median (range) }\end{array}$ & $\begin{array}{c}\text { n / Mean } \pm \text { SD / } \\
\text { Median (range) }\end{array}$ & $\begin{array}{l}\text { n / Mean } \pm \text { SD / } \\
\text { Median (range) }\end{array}$ \\
\hline Age (years) ${ }^{1}$ & $23.1(4.82)^{\mathrm{a}}$ & $36.9(3.04)^{a}$ & $24.8(3.65)$ & $30.8(6.89)$ \\
\hline$<20$ & 3 & 0 & 0 & 1 \\
\hline $20-34$ & 7 & 2 & 10 & 5 \\
\hline$\geq 35$ & 0 & 8 & 0 & 4 \\
\hline Gravida $^{2}$ & $1(1-2)^{b}$ & $2(1-4)^{b, c}$ & $2(1-3)$ & $1(1-3)^{c}$ \\
\hline Primigravida & 7 & 2 & 3 & 6 \\
\hline Multigravida & 3 & 8 & 7 & 4 \\
\hline UK (week) $)^{3}$ & 33 (29-33) & 33 (26-33) & $38(35-40)$ & $37(35-39)$ \\
\hline$<34$ & 10 & 10 & 0 & 0 \\
\hline$>34$ & 0 & 0 & 10 & 10 \\
\hline BMI $\left(\mathrm{kg} / \mathrm{m}^{2}\right)^{2}$ & $24.7(22.3-36.8)$ & $24.6(22.4-37.2)$ & $23.9(21.3-34.2)^{d}$ & $32.8(23.8-40.1)^{d}$ \\
\hline Normal & 7 & 4 & 6 & 1 \\
\hline Overweight & 0 & 2 & 2 & 1 \\
\hline Obesity I & 2 & 3 & 2 & 6 \\
\hline Obesity II & 1 & 1 & 0 & 1 \\
\hline Obesity III & 0 & 0 & 0 & 1 \\
\hline \multicolumn{5}{|l|}{ Type of Delivery ${ }^{2}$} \\
\hline $\mathrm{SC}$ & 1 & 8 & 4 & 4 \\
\hline Spontaneous & 9 & 2 & 5 & 4 \\
\hline Extraction Vacuum & 0 & 0 & 1 & 2 \\
\hline \multicolumn{5}{|c|}{ Maternal Complication ${ }^{3}$} \\
\hline Lung Edema & 0 & 2 & 0 & 0 \\
\hline Eclampsia & 0 & 2 & 0 & 1 \\
\hline HELLP Syndrome & 0 & 1 & 0 & 0 \\
\hline
\end{tabular}

*Significant value $p<0.05$. ' One way Anova, ${ }^{2}$ Kruskal-Wallis, ${ }^{3}$ Mann-Whitney. a: comparison between a-a $(p<0.001)$; b: comparison between $\mathrm{b}-\mathrm{b}(p=0.012)$; : comparison between $\mathrm{c}-\mathrm{c}(\mathrm{p}=0.041)$; $\mathrm{d}$ : comparison between $\mathrm{d}-\mathrm{d}(p=0.010)$.

EO-NP $(p<0.001)$, the number of gravidity between the EOPE $v s$. EO-NP $(p=0.012)$ and EO-PE $v s$. LO-PE $(p=0.041)$. BMI between LO-PE vs. LO-NP was also significantly different $(p=0.010)$, while other parameters did not show any significant different.

\section{Plasma Umbilical Cord Hemeoxygenase-1 (HO-1)}

Plasma umbilical cord HO-1 level of EO-PE $(0.96 \pm 0.37 \mathrm{ng} /$ $\mathrm{mL})$ was significantly lower compared to EO-NP $(2.43 \pm 0.58$ $\mathrm{ng} / \mathrm{mL} ; p<0.001)$, and LO-PE $(2.18 \pm 1.07 \mathrm{ng} / \mathrm{mL} ; p=0.034)$. While plasma umbilical cord HO-1 in LO-PE was not different with LO-NP, although tend to be lower (Figure 1, Table 2). The lowest HO-1 level was found in EO-PE group $(0.55 \mathrm{ng} / \mathrm{mL})$, and the highest was in LO-NP $(3.86 \mathrm{ng} / \mathrm{mL})$.

\section{Neonatal Outcome}

In term of neonatal outcome, there were significant differences in Apgar scores $(p=0.008)$, baby birth weight $(p<0.001)$, and length of stay $(p=0.001)$, between EO-PE and LO-PE (Table 3). And the length of stay of LO-PE was longer than LO-NP ( $3 v s .2 .5 ; p=0.038)$. But there were no significant differences in apgar score and baby birth weight between EO-PE vs. EO-NP, and LO-PE vs. LO-NP.

The assessment of neonatal composite morbidity includes low Apgar score (Apgar score <3), low birth weight (birth weight $<2000$ grams), length of stay $>5$ days, complications in newborns at hospital, such as respiratory distress syndrome, neonatal jaundice, and neonatal death. Any occurrence of these variable on baby indicating 


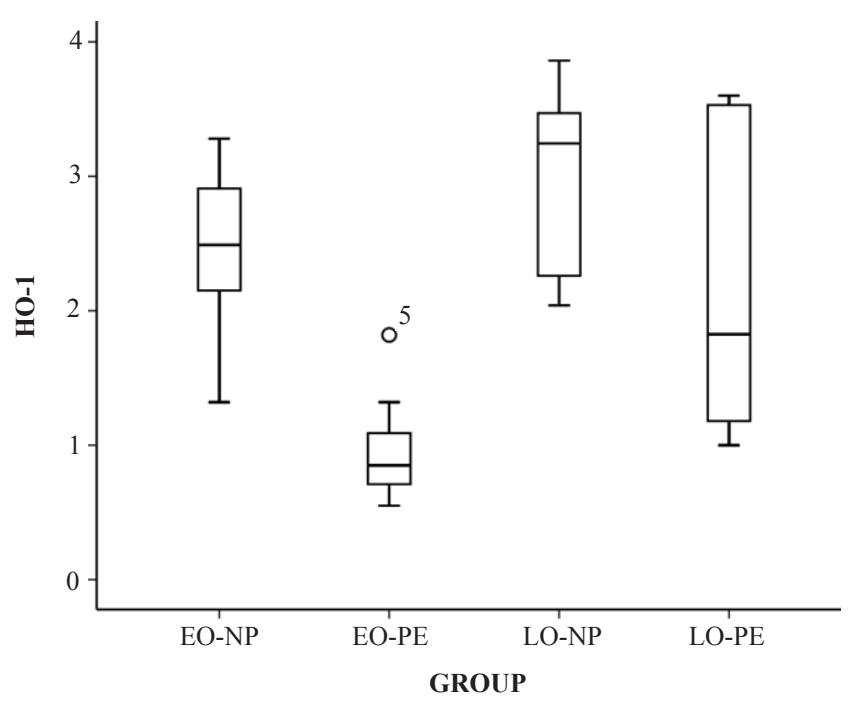

Figure 1. Plasma umbilical cord HO-1 level in EO-NP, EO-PE, LO-NP and LO-PE.

positive neonatal composite morbidity. The EO-PE had more neonatal composite morbidity than LO-PE and EO$\mathrm{NP}$, indicating that the outcomes of newborns in EO-PE was worse compared to the LO-PE $(p=0.003)$ and EO-NP $(p=0.033)$. While the composite morbidity in LO-PE was not different with LO-NP $(p=0.211)$ (Table 4$)$. Based on further statistical analysis using the contingency coefficient test, there was a significant positive correlation of neonatal composite morbidity with EO-PE, with medium strength $(p=0.010, \mathrm{r}=0.500)$. It means that the occurrence of EO-PE increase the risk of composite neonatal morbidity.

Based on the eta association test, a positive correlation was found to be very strong between plasma umbilical cord HO-1 level and neonatal composite morbidity $(p<0.001$, $\mathrm{r}=0.974$ ). Low levels of plasma umbilical cord HO-1 correlate with worse neonatal outcome.

\section{Discussion}

This study support the hypothesis that EO-PE has a different pathogenesis compared to LO-PE, which is involvement of placenta. Plasma umbilical cord HO-1 can represent the level of placental HO-1, and the lower placental HO-1 is correlated with abnormal placental blood vessel development and inadequate vascularization. Our study reveal that in EO-PE group the plasma umbilical cord HO-1 level was lower than LO-PE and EO-NP. While HO-1 level in LOPE was not different with LO-NP. Many studies (animal model and human) had already proven that deficiency of placental HO-1 were correlated with smaller spiral arteries, inadequate placental vascularization, different placental angiogenic profile, and significantly higher blood pressure, which is the main feature of placental preeclampsia. ${ }^{10-12}$ Placental tissue of preeclampsia pregnant women has already proven to have a lower expression of HO-1, lower mRNA HO-1, and lower HO-1 protein level compared to normal pregnancy. ${ }^{6,13,14}$

Deficiency HO-1 may also shift the balance toward anti angiogenesis dominant, which result in endothelial cell damage through its relation with soluble FMS like tyrosine kinase-1 (sFlt-1) and soluble endoglin (sEng). ${ }^{15}$ Cudmore, et al., showed that induction of HO-1 in human umbilical endothelial vein endothelial cells (HUVECs) inhibit the release of sFlt-1 and sEng. ${ }^{16,17}$ Circulating sFlt-1 and sEng are the two main factors in the development of clinical syndrome of preeclampsia. Our study result indicating that in EO-PE (but not in LO-PE) the placenta was involved, reflected by the lower level of plasma HO-1. This placental vascular involvement in EO-PE will decrease uteroplacental blood flow, lead to uteroplacental insufficiency and correlate with poor pregnancy outcome. ${ }^{18}$ While LO-PE is associated with normal placental vascular development, normal uteroplacental perfusion, normal placental volume and better pregnancy outcome., ${ }^{2,19}$ Our study support this hypothesis with the finding that neonatal outcome and composite morbidity in EO-PE was worse compare to the LO-PE and EO-NP. Baby delivered from EO-PE woman had lower Apgar score, lower birth weight, and longer length of stay compared to LO-PE. Since neonatal morbidity also

Table 2. Plasma levels of umbilical cord HO-1.

\begin{tabular}{llccc}
\hline \multirow{2}{*}{ Variable } & \multicolumn{1}{c}{ Group } & Early Onset & Late Onset & \\
\cline { 3 - 4 } & & $\begin{array}{c}\text { Mean } \pm \text { SD } \\
(\mathbf{n g} / \mathbf{m L})\end{array}$ & $\begin{array}{c}\text { Mean } \pm \text { SD } \\
(\mathbf{n g} / \mathbf{m L})\end{array}$ & $p$-value \\
\hline \multirow{2}{*}{$\begin{array}{l}\text { Hemeoksigenase }-1 \\
(\text { HO- } 1)\end{array}$} & Control & $2.43 \pm 0.58$ & $3.02 \pm 0.64$ & - \\
& Severe Preeclampsia & $0.96 \pm 0.37$ & $2.18 \pm 1.07$ & $0.034^{*}$ \\
& $p$-value & $<0.001^{*}$ & 0.277 & - \\
\hline
\end{tabular}

*Significant value $\mathrm{p}<0.05$, tested with ANOVA. 
Table 3. Neonatal outcome.

\begin{tabular}{|c|c|c|c|c|}
\hline \multirow{2}{*}{ Variable } & \multirow{2}{*}{ Group } & Early Onset & Late Onset & \multirow{2}{*}{$p$-value } \\
\hline & & Median (range) & Median (range) & \\
\hline \multirow{3}{*}{ Apgar score $^{2}$} & Control & $6(6-8)$ & $8(7-8)$ & - \\
\hline & Severe Preclampsia & $5(3-8)$ & $7.5(6-8)$ & $0.008 *$ \\
\hline & $p$-value & 0.149 & 0.313 & - \\
\hline \multirow{3}{*}{$\begin{array}{l}\text { Birth Weight } \\
\text { (grams) }\end{array}$} & Control & $2095(1300-2300)$ & $3145(2590-3660)$ & - \\
\hline & Severe Preclampsia & $1900(1000-2240)$ & $2985(2510-3220)$ & $<0.001 *$ \\
\hline & $p$-value & 0.495 & 0.241 & - \\
\hline \multirow{3}{*}{$\begin{array}{l}\text { Length of stay }{ }^{2} \\
\text { (days) }\end{array}$} & Control & $7.5(2-28)$ & $2.5(1-3)$ & - \\
\hline & Severe Preclampsia & $17(5-30)$ & $3(2-7)$ & $0.001 *$ \\
\hline & $p$-value & 0.079 & $0.038 *$ & - \\
\hline
\end{tabular}

*Significant value $p<0.05$. ${ }^{1}$ One way Anova, ${ }^{2}$ Kruskal-Wallis, ${ }^{3}$ Mann-Whitney.

closely related to gestational age at the time of delivery, more severe form of EO-PE often necessitate iatrogenic preterm delivery, which result in prematurity. Prematurity will directly increase neonatal morbidity (respiratory distress syndrome, neonatal jaundice) and mortality. Our finding is in line with many recent studies which stated that neonatal outcome in EO-PE was worse compared to LO-PE. ${ }^{19-21}$ And also the neonatal outcome in LO-PE was not different with the LO-NP, indicating relatively normal placental development and uteroplacental perfusion in this type of preeclampsia.

This study also reveal a strong correlation between plasma umbilical cord HO-1 level with neonatal composite morbidity. It means that low umbilical cord HO-1 level is correlated with poor neonatal outcome. Many studies had proven that deficiency HO-1 in placenta and circulation was correlated with poor pregnancy outcome, such as miscarriage, intra uterine growth restriction, intra uterine fetal death, and preterm labour. ${ }^{22-27}$ This might be explained by an unopposed free fetal heme on deficient HO-1 placenta that stimulates cell damage, subsequently lead to abnormal placentation. ${ }^{27}$ But as far as we know, this was the first study to evaluate the relationship between placental HO-1 and neonatal outcome. The strong relationship between low level of placental plasma HO-1 and neonatal composite morbidity (especially in EO-PE) could provide clue about the role of placental HO-1 deficiency in the development of more severe type of preeclampsia with abnormal placental development and poor neonatal outcome. Defficiency of placental HO-1 was correlated with increased level of sFlt-1 and sEng, which associated with clinical severity of preeclampsia. ${ }^{4}$ Study on animal model showed that induction of HO-1 inhibit sFlt-1 and sEng level, increased VEGF level, improved endothelial fuction, and decreased blood pressure. ${ }^{16,17,28}$

Our study result indicate that in the future, HO-1 inducer (statin) may be used to protect the normal placental vascular development in pregnancy, prevent placental insufficiency syndrome such as preeclampsia (especially the early type), and improve the neonatal outcome.

Table 4. Composite neonatal morbidity.

\begin{tabular}{lcccc}
\hline \multicolumn{1}{c}{ Variables } & EO-NP & EO-PE & LO-NP & LO-PE \\
\hline Apgar score $<3$ & 0 & 2 & 0 & 0 \\
Birth Weight $<$ 2000 grams & 2 & 5 & 0 & 0 \\
Length of Stay $>$ 5 days & 5 & 7 & 0 & 2 \\
Respiratory Distress Syndrome & 1 & 3 & 0 & 0 \\
Neonatal Jaundice & 2 & 7 & 0 & 3 \\
Neonatal Death & 1 & 3 & 0 & 0 \\
Neonatal Composite Morbidity & 5 & 10 & 0 & 3 \\
\hline
\end{tabular}




\section{Conclusion}

This study reveal that defficiency placental HO-1 is correlated with poor neonatal outcome (especially in EO$\mathrm{PE)}$ via abnormal placental vascular development. HO-1 inducer (statin) should be consider as a new approach in the future to protect vascular placental insufficiency, prevent subsequent preeclampsia syndrome, and improve neonatal outcome.

\section{References}

1. Wang K, Ahmad S, Cai M, Rennie J, Fujisawa T, Crispi F, et al. Dysregulation of hydrogen sulfide producing enzyme cystathioninelyase contributes to maternal hypertension and placental abnormalities in preeclampsia. Circulation. 2013; 127(25): 2514-22.

2. Sulistyowati S. Early and Late Onset Preeclampsia: What did really matter?. J Gynecol Women's Health. 2017; 5(4): 555670. doi: 10.19080/jgwh.2017.05.555670.

3. Chaiworapongsa T, Chaemsaithong P, Yeo L, Romero R. Preeclampsia part 1: current understand of its pathophysiology. Nat Rev Nephrol. 2014; 10: 466-80.

4. Ahmed A, Ramma W. Unravelling the theories of preeclampsia: are the protective pathways the new paradigma. Br J Pharmacol. 2015; 172(6): 1574-86.

5. Zhao H, Wong RJ, Kalish FS, Nayak NR, Stevenson DK. Effect of heme oxygenase-1 deficiency on placental development. Placenta. 2009; 30(10): 861-8.

6. Ahmed A, Rahman M, Zhang X, Acevedo CH, NIjjar S, Rushton I, et al. Induction of placental heme oxygenase-1 is protective against $\mathrm{TNF} \alpha$-induced cytotoxicity and promotes vessel relaxation. Mol Med. 2000; 6(5): 391-409.

7. Tranquilli AL, Dekker G, Magee L, Roberts J, Sibai BM, Steyn $\mathrm{W}$, et al. The classification, diagnosis, and management of the hypertensive disorders of pregnancy: A revised statement from the ISSHP. Pregnancy Hypertens. 2014; 4(2): 97-104.

8. Vitaratos N, Papakonstantinou K, Deliveliotou A, Economou E, Panoulis C, Hassiakos D, et al. Antepartum and postpartum serum heme oxygenase-1 levels in preeclamptic and normotensive pregnant women. In Vivo. 2011; 25(3): 445-50.

9. Committee on Obstetric Practice American Academy of Pediatrics Committee on Fetus and Newborn. Committee Opinion No. 644: The Apgar Score. Obstet Gynecol. 2015; 126(4): e52-5.

10. Mclean M, Bowman M, Clifton V, Smith R, Grossman AB. Expression of the heme oxygenase - carbon monoxide signalling system in human placenta. J Clin Endocrinol Metab. 2000; 85(6): 2345-9.

11. Zhao H, Wong RJ, Doyle TC, Nayak N, Vreman HJ, Contag CH, et al. Regulation of maternal and fetal hemodynamics by heme oxygenase in mice. Biol Reprod. 2008; 78(4): 744-51.

12. Zhao H, Azuma J, Kalish F, Wong RJ, Stevenson DK. Maternal heme oxygenase 1 regulates placental vasculature development via angiogenic factors in mice. Biol Reprod. 2011; 85(5): 1005-12.
13. Padmini E, Lavanya D. HIF- $1 \alpha$, TNF- $\alpha$, and HO-1 modulation in placental explants during preeclampsia. Int J Res Chem Environ. 2014; 4(2): 85-92.

14. Farina A, Sekizawa A, De Sanctis P, Purwosunu Y, Okai T, Cha DH. Gene expression in chorionic villous samples at 11 weeks gestation from women destined to develop preeclampsia. Prenat Diagn. 2008; 28(10): 956-61.

15. Levytska K, Kingdom J, Baczyk D, Drewlo S. Heme oxygenase-1 in placental development and pathology. Placenta. 2013; 34(4): 291-8.

16. Cudemore M, Ahmad S, Al-Ani B, Fujisawa T, Coxall H, Chudasama $\mathrm{K}$, et al. Negative regulation of soluble Flt-1 and soluble endoglin release by heme oxygenase-1. Circulation. 2007; 115(13): 1789-97.

17. Cudemore MJ, Ramma W, Cai M, Fujisawa T, Ahmad S, Al-Ani B, et al. Resveratrol inhibits the release of soluble fms-like tyrosine kinase (sFlt-1) from human placenta. Am J Obstet Gynecol 2012; 206(3): 253.e10-5.

18. Than NG, Vaisbuch, E, Kim CJ. Early-onset preeclampsia and HELLP Syndrome: an Overview. In: Handbook of Growth Monitoring in Health and Disease. New York: Springer Science; 2011. p.1867-91.

19. Madazli R, Yuksel MA, Imamoglu M, Tuten A, Oncul M, Aydin B, et al. Comparison of clinical and perinatal outcomes in early and late onset preeclampsia. Arch Gynecol Obstet. 2014; 290(1): 53-7.

20. Bozdag H, Ogutcuoglu FBG, Guzin K, Kabaca Kilic SR, Duran EA, Topdagi Aydin I, et al. The frequency and fetomaternal outcomes of early and late onset preeclampsia: The experience of a single tertiary center in bustling metroplosi of Turkey; Istanbul. Med Med J. 2015; 30(4): 163-9.

21. Aldika Akbar MI, Herdiyantini M, Aryananda RA, Cininta N, Wardhana MP, Gumilar KE, et al. Serum heme oxygenase 1 (HO1), soluble FMS like tyrosine kinase (sFlt-1) level, and neonatal outcome in early onset, late onset preeclampsia and normal pregnancy. Hypertens Pregnancy. 2018; 37(4): 175-81.

22. Maroti Z, Katona M, Orvos H, Nemeth I, Farkas I, Turi S. Heme oxygenase-1 expression in premature and mature neonates during the first week of life. Eur J Pediatr. 2007; 166(10): 1033-8.

23. Barber A, Robson SC, Myatt L, Bulmer JN, Lyall F. Heme oxygenase expression in human placenta and placenta bed: reduced expression of placenta endothelial HO-2 in preeclampsia and fetal growth restriction. FASEB J. 2001; 15(7): 1158-68.

24. Dennery PA. Signaling fuction of heme oxygenase proteins. Antioxid Redox Signal. 2014; 20(11): 1743-53.

25. Gonzalez S, Perez MJ, Perazzo JC, Tomaro Ml. Antioxidant role of heme oxygenase-1 in prehepatic portal hypertensive rats. World J Gastroenterol. 2006; 12(26): 4149-55.

26. Wong RJ, Zhao H, Stevenson DK. A deficiency in heme oxygenase-1 induces foetal growth restriction by placental vasculature defects. Acta Peaeditrica. 2012; 101(8): 827-34.

27. Zenclussen ML, Linzke N, Schumacher A, Fest S, Meyer N, Cassalis $\mathrm{PA}$, et al. Heme oxygenase- 1 is critically involved in placentation, spiral artery remodeling, and blood pressure regulation during murine pregnancy. Front Pharmacol. 2015; 5: 291. doi: 10.3389/ fphar.2014.00291.

28. George EM, Colson D, Dixon J, Palei AC, Granger JP. Heme oxygenase- 1 attenuates hypoxia-induced sFlt- 1 and oxidative stress in placental villi through its metabolic products Co and bilirubin. In J Hypertens. 2012; 2012: 486053. doi: 10.1155/2012/486053. 